Acta Crystallographica Section E

Structure Reports

Online

ISSN 1600-5368

\section{Bis(7-amino-2,4-dimethyl-1,8-naphthyri- dine)dinitratocadmium(II)}

\section{Shou-Wen Jin,* Qi-Jun Zhao, Xian-Gang Qian, Ru-Xiang Chen and Yan-Fen Shi}

Faculty of Science, Zhejiang Forestry University, Lin'An 311300, People's Republic of China

Correspondence e-mail: jinsw@zjfc.edu.cn

Received 15 November 2007; accepted 23 November 2007

Key indicators: single-crystal X-ray study; $T=298 \mathrm{~K}$; mean $\sigma(\mathrm{C}-\mathrm{C})=0.006 \AA$; $R$ factor $=0.035 ; w R$ factor $=0.081 ;$ data-to-parameter ratio $=12.8$.

In the title compound, $\left[\mathrm{Cd}\left(\mathrm{NO}_{3}\right)_{2}\left(\mathrm{C}_{10} \mathrm{H}_{11} \mathrm{~N}_{3}\right)_{2}\right]$, two naphthyridine ring systems are coordinated to the $\mathrm{Cd}$ ion through the two $\mathrm{N}$ atoms in a bidentate chelating mode, whereas the remaining coordination sites are occupied by two $\mathrm{O}$ atoms from two different nitrate groups to complete the octahedral geometry. Intermoleular $\mathrm{N}-\mathrm{H} \cdots \mathrm{O}$ hydrogen bonds link the molecules to form a one-dimensionnal sheet parallel to the $a c$ plane. Weak slipped $\pi-\pi$ stacking involving the naphthyridine ring systems stabilizes the structure.

\section{Related literature}

For related literature, see: Bayer (1979); Che et al. (2001); Gavrilova \& Bosnich (2004); Jin et al. (2007); Kukrek et al. (2006); Mintert \& Sheldrick (1995a,b); Oskui \& Sheldrick (1999); Oskui, Mintert \& Sheldrick (1999).

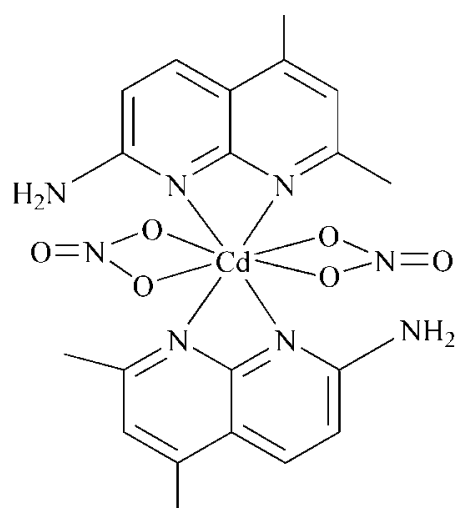

\section{Experimental}

\section{Crystal data}

$\left[\mathrm{Cd}\left(\mathrm{NO}_{3}\right)_{2}\left(\mathrm{C}_{10} \mathrm{H}_{11} \mathrm{~N}_{3}\right)_{2}\right]$

$M_{r}=582.86$

Triclinic, $P \overline{1}$

$$
\begin{aligned}
& \alpha=95.497(3)^{\circ} \\
& \beta=95.224(3)^{\circ} \\
& \gamma=116.865(3)^{\circ} \\
& V=1179.8(5) \AA^{3} \\
& Z=2
\end{aligned}
$$

Data collection

Bruker SMART diffractometer Absorption correction: multi-scan (SADABS; Sheldrick, 1996) $T_{\min }=0.667, T_{\max }=0.751$

\section{Refinement}

$R\left[F^{2}>2 \sigma\left(F^{2}\right)\right]=0.035$

$w R\left(F^{2}\right)=0.081$

$S=1.09$

4101 reflections
Mo $K \alpha$ radiation

$\mu=0.98 \mathrm{~mm}^{-1}$

\begin{tabular}{|c|c|c|c|c|}
\hline$D-\mathrm{H} \cdots A$ & $D-\mathrm{H}$ & $\mathrm{H} \cdots A$ & $D \cdots A$ & $D-\mathrm{H} \cdots A$ \\
\hline $\mathrm{N} 3-\mathrm{H} 3 A \cdots \mathrm{O} 2$ & 0.86 & 2.23 & $3.033(4)$ & 155 \\
\hline $\mathrm{N} 3-\mathrm{H} 3 B \cdots \mathrm{O} 5^{\mathrm{i}}$ & 0.86 & 2.38 & 3.161 (4) & 151 \\
\hline $\mathrm{N} 6-\mathrm{H} 6 A \cdots \mathrm{O} 5$ & 0.86 & 2.11 & $2.902(4)$ & 153 \\
\hline $\mathrm{N} 6-\mathrm{H} 6 B \cdots \mathrm{O} 3^{\mathrm{ii}}$ & 0.86 & 2.18 & $2.979(4)$ & 154 \\
\hline
\end{tabular}

$T=298$ (2) K

$0.45 \times 0.37 \times 0.31 \mathrm{~mm}$

Table 1

Hydrogen-bond geometry $\left(\AA{ }^{\circ}\right)$.

Symmetry codes: (i) $x+1, y, z$; (ii) $-x,-y+1,-z$.

Table 2

Main $\pi-\pi$ interactions $\left(\AA,{ }^{\circ}\right)$.

$\alpha$ is the dihedral angle between the planes. DCC is the length of the CC vector (centroid to centroid). $\tau$ is the angle(s) subtended by the plane(s) normal to $\mathrm{CC}$ (offset angle). $C g 1$ is the centroid of ring N1/C1/C5-C8; $C g 2$ is the centroid of ring $\mathrm{N} 2 / \mathrm{C} 1 / \mathrm{C} 5-\mathrm{C} 2$.

\begin{tabular}{lllll}
\hline Centroid 1 & Centroid 2 & $\alpha$ & DCC & $\tau$ \\
\hline$C g 1$ & $C g 2^{\text {iii }}$ & 1.32 & $3.862(2)$ & 26 \\
$C g 2$ & $C g 2^{\text {iii }}$ & 0.0 & $3.823(2)$ & 25 \\
\hline
\end{tabular}

Symmetry code: (iii) $-x+1,-y+1,-z+1$.

Data collection: SMART (Bruker, 1999); cell refinement: SAINT (Bruker, 1999); data reduction: $S A I N T$; $\operatorname{program}(\mathrm{s})$ used to solve structure: SHELXS97 (Sheldrick, 1997); program(s) used to refine structure: SHELXL97 (Sheldrick, 1997); molecular graphics: ORTEPIII (Burnett \& Johnson, 1996), ORTEP-3 for Windows (Farrugia, 1997) and PLATON (Spek, 2003); software used to prepare material for publication: SHELXL97.

The authors thank the Zhejiang Forestry University Science Foundation for financial support.

Supplementary data and figures for this paper are available from the IUCr electronic archives (Reference: DN2283).

\title{
References
}

Bayer, J. W. (1979). US Patent 4169092.

Bruker (1999). SMART (Version 5.611) and SAINT (Version 6.02a). Bruker AXS Inc., Madison, Wisconsin, USA.

Burnett, M. N. \& Johnson, C. K. (1996). ORTEPIII. Report ORNL-6895. Oak Ridge National Laboratory, Tennessee, USA.

6136 measured reflections 4101 independent reflections 3346 reflections with $I>2 \sigma(I)$

320 parameters

$\Delta \rho_{\max }=0.67{\mathrm{e} \AA^{-3}}^{-3}$

$\Delta \rho_{\min }=-0.36 \mathrm{e}^{-3}$ 


\section{metal-organic compounds}

Che, C. M., Wan, C. W., Hoa, K. Y. \& Zhou, Z. Y. (2001). New J. Chem. 25, 6365.

Farrugia, L. J. (1997). J. Appl. Cryst. 30, 565.

Gavrilova, A. L. \& Bosnich, B. (2004). Chem. Rev. 104, 349-383.

Jin, S. W., Liu, B. \& Chen, W. Z. (2007). Chin. J. Struct. Chem. 26, 287-290.

Kukrek, A., Wang, D., Hou, Y. J., Zong, R. F. \& Thummel, R. (2006). Inorg. Chem. 45, 10131-10137.

Mintert, M. \& Sheldrick, W. S. (1995a). Inorg. Chim. Acta, 236, 13-20.
Mintert, M. \& Sheldrick, W. S. (1995b). J. Chem. Soc. Dalton Trans. pp. 2663 2669.

Oskui, B., Mintert, M. \& Sheldrick, W. S. (1999). Inorg. Chim. Acta, 287, 72-81. Oskui, B. \& Sheldrick, W. S. (1999). Eur. J. Inorg. Chem. pp. 1325-1328.

Sheldrick, G. M. (1996). SADABS. University of Göttingen, Germany.

Sheldrick, G. M. (1997). SHELXS97 and SHELXL97. University of Göttingen, Germany.

Spek, A. L. (2003). J. Appl. Cryst. 36, 7-13. 


\section{supporting information}

Acta Cryst. (2008). E64, m54-m55 [https://doi.org/10.1107/S160053680706271X]

Bis(7-amino-2,4-dimethyl-1,8-naphthyridine)dinitratocadmium(II)

Shou-Wen Jin, Qi-Jun Zhao, Xian-Gang Qian, Ru-Xiang Chen and Yan-Fen Shi

\section{S1. Comment}

Molecular structures and chemical properties of transition metal complexes of 1,8-naphthyridine (napy) and its derivatives have received much attention(Kukrek et al., 2006; Che et al., 2001), because the ligands can link to metals with several coordination modes such as monodentate, chelating bidentate, and dinuclear bridging binding fashion (Gavrilova \& Bosnich, 2004). 5,7-dimethyl-1,8-naphthyridin-2-amine $(L)$ is a potentially tridentate ligand and is capable of linking two to four metal atoms together to form metal aggregates (Oskui et al., 1999; Mintert \& Sheldrick, 1995a; Oskui \& Sheldrick, 1999; Mintert \& Sheldrick, 1995b). The coordination chemistry of 5,7-dimethyl-1,8-naphthyridine-2amine has not been well studied before although a series of transiton metal complex $\left(M_{(L)} 2_{\mathrm{C} 1}\right)$ were once described in a US patent (Bayer, 1979). As an extension of our study on naphthyridine coordination chemistry(Jin et al., 2007), herein the title complex $\left[\mathrm{Cd}(L)_{2}\left(\mathrm{NO}_{3}\right)_{2]}\right.$ is reported.

In the title compound, two naphthyridine are coordinated to the $\mathrm{Cd}$ ion through two nitrogen atoms in bidentate chelating mode whereas the remaining coordination sites are occupied by two oxygen atoms from two different nitrates (Fig. 1). The two nitrate ligands display a dissymetric chelating mode with $\mathrm{Cd}-\mathrm{O}$ distances of 2.357 (4) and 2.718 (4). The remaining distances are within the usual range. The two naphthyridine rings were almost perpendicular to each other making dihedral angle of $80.22(7)^{\circ}$. The two chelating $\mathrm{Cd}-\mathrm{O}-\mathrm{N}-\mathrm{O}$ group make dihedral angle of $81.21(12)^{\circ}$. Intermoleular $\mathrm{N}-\mathrm{H} \cdots \mathrm{O}$ hydrogen bonds link the molecules to form a one dimensionnal sheet parallel to the $a$ axis (Table 1, Fig. 2). Weak slippest $\pi-\pi$ stackings involving the naphthyridine rings stabilize the structure (Table 2).

\section{S2. Experimental}

All reagents and solvents were used as obtained without further purification. The $\mathrm{CHN}$ elemental analyses were performed on a Perkin-Elmer model 2400 elemental analyzer. A solution of cadmium nitrate tetrahydrate (31.4 mg, 0.1 $\mathrm{mmol})$ in methanol $(3 \mathrm{ml})$ was added to $L(52.2 \mathrm{mg}, 0.3 \mathrm{mmol})$ in methanol $(10 \mathrm{ml})$ to give a colorless solution. The methanol solution was filtered. The solution was left standing at room temperature for several days, colorless block crystals were isolated. Yield: $41 \mathrm{mg}, 70.3 \%$. Anal. Calcd. for $\mathrm{C}_{20} \mathrm{H}_{22} \mathrm{Cd}_{\mathrm{N}} 8_{0} 6$ : C, 41.18; H, 3.77; N, 19.22. Found: C, 41.14; H, 3.72; N, 19.18.

\section{S3. Refinement}

All $\mathrm{H}$ atoms attached to $\mathrm{C}$ atoms and $\mathrm{N}$ atom were fixed geometrically and treated as riding with $\mathrm{C}-\mathrm{H}=0.93 \AA$ (aromatic) or $0.96 \AA$ (methyl) and $\mathrm{N}-\mathrm{H}=0.86 \AA$ with $U_{\text {iso }}(\mathrm{H})=1.2 U_{\text {eq }}($ Caromatic or $\mathrm{N})$ or $U_{\text {iso }}(\mathrm{H})=1.5 U_{\text {eq }}($ Cmethyl). 


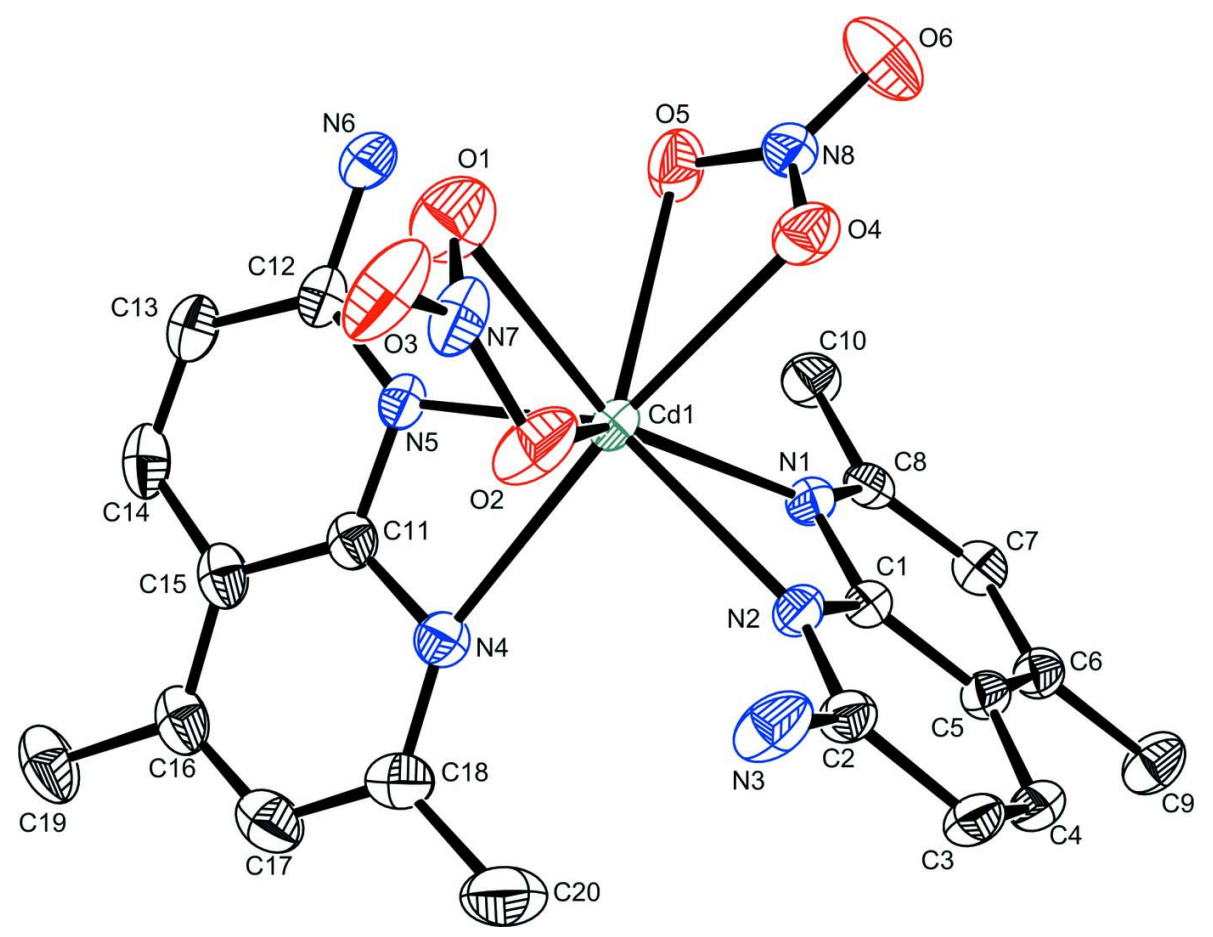

Figure 1

Molecular view of the title compound, showing the atom-numbering scheme. Displacement ellipsoids are drawn at the $30 \%$ probability level. $\mathrm{H}$ atoms have been omitted for clarity. 


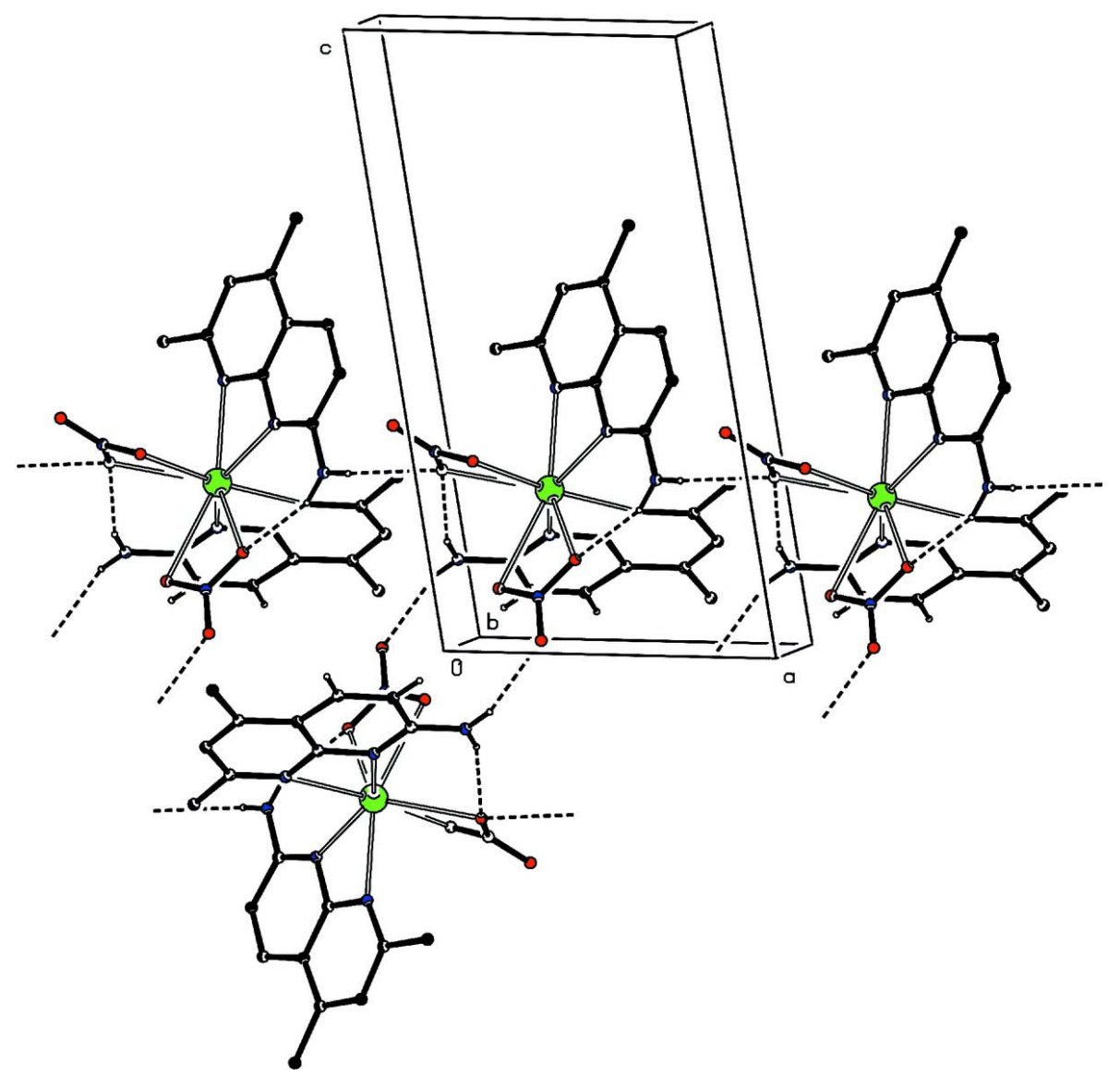

Figure 2

Partial packing view showing the $\mathrm{N}-\mathrm{H} \cdots \mathrm{O}$ hydrogen bonds. Hydrogen bonds are shown as dashed lines. $\mathrm{H}$ atoms not involved in hydrogen bondings have been omitted for clarity.

Dinitratobis(7-amino-2,4-dimethyl-1,8-naphthyridine)cadmium(II)

\section{Crystal data}

$\left[\mathrm{Cd}\left(\mathrm{NO}_{3}\right)_{2}\left(\mathrm{C}_{10} \mathrm{H}_{11} \mathrm{~N}_{3}\right)_{2}\right]$

$M_{r}=582.86$

Triclinic, $P \overline{1}$

Hall symbol: -P 1

$a=9.308$ (2) $\AA$

$b=9.584(2) \AA$

$c=15.067$ (4) $\AA$

$\alpha=95.497(3)^{\circ}$

$\beta=95.224(3)^{\circ}$

$\gamma=116.865(3)^{\circ}$

$V=1179.8(5) \AA^{3}$

\section{Data collection}

Bruker SMART

diffractometer

Radiation source: fine-focus sealed tube

Graphite monochromator

$\varphi$ and $\omega$ scans
$Z=2$

$F(000)=588$

$D_{\mathrm{x}}=1.641 \mathrm{Mg} \mathrm{m}^{-3}$

Mo $K \alpha$ radiation, $\lambda=0.71073 \AA$

Cell parameters from 2793 reflections

$\theta=2.4-26.1^{\circ}$

$\mu=0.98 \mathrm{~mm}^{-1}$

$T=298 \mathrm{~K}$

Block, colorless

$0.45 \times 0.37 \times 0.31 \mathrm{~mm}$

Absorption correction: multi-scan

(SADABS; Sheldrick, 1996)

$T_{\min }=0.667, T_{\max }=0.751$

6136 measured reflections

4101 independent reflections 
3346 reflections with $I>2 \sigma(I)$

$R_{\text {int }}=0.019$

$\theta_{\max }=25.0^{\circ}, \theta_{\min }=2.4^{\circ}$

\section{Refinement}

Refinement on $F^{2}$

Least-squares matrix: full

$R\left[F^{2}>2 \sigma\left(F^{2}\right)\right]=0.035$

$w R\left(F^{2}\right)=0.081$

$S=1.09$

4101 reflections

320 parameters

0 restraints

Primary atom site location: structure-invariant direct methods $h=-11 \rightarrow 6$

$k=-11 \rightarrow 11$

$l=-17 \rightarrow 17$

Secondary atom site location: difference Fourier map

Hydrogen site location: inferred from neighbouring sites

$\mathrm{H}$-atom parameters constrained

$w=1 /\left[\sigma^{2}\left(F_{\mathrm{o}}^{2}\right)+(0.037 P)^{2}+0.1524 P\right]$ where $P=\left(F_{\mathrm{o}}^{2}+2 F_{\mathrm{c}}{ }^{2}\right) / 3$

$(\Delta / \sigma)_{\max }=0.004$

$\Delta \rho_{\max }=0.67 \mathrm{e} \AA^{-3}$

$\Delta \rho_{\min }=-0.36$ e $\AA^{-3}$

Special details

Geometry. All e.s.d.'s (except the e.s.d. in the dihedral angle between two 1.s. planes) are estimated using the full covariance matrix. The cell e.s.d.'s are taken into account individually in the estimation of e.s.d.'s in distances, angles and torsion angles; correlations between e.s.d.'s in cell parameters are only used when they are defined by crystal symmetry. An approximate (isotropic) treatment of cell e.s.d.'s is used for estimating e.s.d.'s involving 1.s. planes.

Refinement. Refinement of $F^{2}$ against ALL reflections. The weighted $R$-factor $w R$ and goodness of fit $S$ are based on $F^{2}$, conventional $R$-factors $R$ are based on $F$, with $F$ set to zero for negative $F^{2}$. The threshold expression of $F^{2}>\sigma\left(F^{2}\right)$ is used only for calculating $R$-factors(gt) etc. and is not relevant to the choice of reflections for refinement. $R$-factors based on $F^{2}$ are statistically about twice as large as those based on $F$, and $R$ - factors based on ALL data will be even larger.

Fractional atomic coordinates and isotropic or equivalent isotropic displacement parameters $\left(\AA^{2}\right)$

\begin{tabular}{|c|c|c|c|c|}
\hline & $x$ & $y$ & $z$ & $U_{\text {iso }} * / U_{\text {eq }}$ \\
\hline $\mathrm{Cd} 1$ & $0.32533(3)$ & $0.63678(3)$ & $0.248895(17)$ & $0.04168(11)$ \\
\hline N1 & $0.3847(3)$ & $0.7270(3)$ & $0.41178(18)$ & $0.0381(7)$ \\
\hline N2 & $0.5294(3)$ & $0.6210(3)$ & $0.34613(18)$ & $0.0385(7)$ \\
\hline N3 & $0.6662(4)$ & $0.5103(4)$ & $0.2720(2)$ & $0.0624(10)$ \\
\hline $\mathrm{H} 3 \mathrm{~A}$ & 0.6026 & 0.4943 & 0.2228 & $0.075^{*}$ \\
\hline H3B & 0.7418 & 0.4820 & 0.2719 & $0.075^{*}$ \\
\hline N4 & $0.5509(4)$ & $0.8962(4)$ & $0.2110(2)$ & $0.0448(7)$ \\
\hline N5 & $0.2822(4)$ & $0.8123(3)$ & $0.17237(18)$ & $0.0407(7)$ \\
\hline N6 & $0.0057(4)$ & $0.7107(4)$ & $0.1316(2)$ & $0.0616(9)$ \\
\hline H6A & -0.0097 & 0.6348 & 0.1619 & $0.074 *$ \\
\hline H6B & -0.0763 & 0.7152 & 0.1034 & $0.074^{*}$ \\
\hline N7 & $0.2627(5)$ & $0.4197(4)$ & $0.0808(2)$ & $0.0568(9)$ \\
\hline N8 & $0.0220(4)$ & $0.4282(4)$ & $0.3115(2)$ & $0.0459(8)$ \\
\hline $\mathrm{O} 1$ & $0.1458(4)$ & $0.4451(4)$ & $0.0924(2)$ & $0.0826(10)$ \\
\hline $\mathrm{O} 2$ & $0.3852(4)$ & $0.4845(4)$ & $0.1406(2)$ & $0.0784(10)$ \\
\hline $\mathrm{O} 3$ & $0.2607(4)$ & $0.3373(4)$ & $0.0126(2)$ & $0.0803(10)$ \\
\hline $\mathrm{O} 4$ & $0.1344(3)$ & $0.3951(3)$ & $0.29808(18)$ & $0.0550(7)$ \\
\hline O5 & $0.0207(4)$ & $0.5429(4)$ & $0.27969(18)$ & $0.0622(8)$ \\
\hline O6 & $-0.0832(5)$ & $0.3513(4)$ & $0.3543(2)$ & $0.0927(11)$ \\
\hline $\mathrm{C} 1$ & $0.5092(4)$ & $0.6900(4)$ & $0.4236(2)$ & $0.0342(8)$ \\
\hline $\mathrm{C} 2$ & $0.6476(4)$ & $0.5792(4)$ & $0.3489(2)$ & $0.0395(8)$ \\
\hline $\mathrm{C} 3$ & 0.7515 & $0.6041(4)$ & $0.4305(2)$ & $0.0447(9)$ \\
\hline
\end{tabular}




$\begin{array}{lllll}\text { H3 } & 0.8323 & 0.5722 & 0.4311 & 0.054^{*} \\ \text { C4 } & 0.7316(4) & 0.6744(4) & 0.5071(2) & 0.0427(9) \\ \text { H4 } & 0.8013 & 0.6939 & 0.5603 & 0.051^{*} \\ \text { C5 } & 0.6056(4) & 0.7189(4) & 0.5074(2) & 0.0361(8) \\ \text { C6 } & 0.5702(4) & 0.7900(4) & 0.5826(2) & 0.0403(8) \\ \text { C7 } & 0.4400(5) & 0.8216(4) & 0.5692(2) & 0.0449(9) \\ \text { H7 } & 0.4116 & 0.8652 & 0.6181 & 0.054^{*} \\ \text { C8 } & 0.3495(4) & 0.7898(4) & 0.4836(2) & 0.0386(8) \\ \text { C9 } & 0.6701(5) & 0.8290(5) & 0.6740(2) & 0.0586(1) \\ \text { H9A } & 0.6225 & 0.8677 & 0.7177 & 0.088^{*} \\ \text { H9B } & 0.7791 & 0.9088 & 0.6724 & 0.088^{*} \\ \text { H9C } & 0.6725 & 0.7356 & 0.6902 & 0.088^{*} \\ \text { C10 } & 0.2099(5) & 0.8259(5) & 0.4700(3) & 0.0533(10) \\ \text { H10A } & 0.1851 & 0.8294 & 0.4073 & 0.080^{*} \\ \text { H10B } & 0.2383 & 0.9266 & 0.5047 & 0.080^{*} \\ \text { H10C } & 0.1165 & 0.7451 & 0.4893 & 0.080^{*} \\ \text { C11 } & 0.4365(5) & 0.9236(4) & 0.1670(2) & 0.0406(8) \\ \text { C12 } & 0.1564(5) & 0.8210(5) & 0.1283(2) & 0.0470(9) \\ \text { C13 } & 0.1818(6) & 0.9478(5) & 0.0788(3) & 0.0560(11) \\ \text { H13 } & 0.0928 & 0.9539 & 0.0496 & 0.067^{*} \\ \text { C14 } & 0.3334(6) & 1.0585(5) & 0.0741(3) & 0.0571(11) \\ \text { H14 } & 0.3489 & 1.1410 & 0.0419 & 0.069^{*} \\ \text { C15 } & 0.4704(5) & 1.0502(4) & 0.1182(2) & 0.0466(9) \\ \text { C16 } & 0.6348(6) & 1.1545(5) & 0.1155(3) & 0.0545(11) \\ \text { C17 } & 0.7501(6) & 1.1242(5) & 0.1602(3) & 0.0623(12) \\ \text { H17 } & 0.8598 & 1.1908 & 0.1592 & 0.075^{*} \\ \text { C18 } & 0.7068(5) & 0.9956(5) & 0.2072(3) & 0.0554(10) \\ \text { C19 } & 0.6828(6) & 1.2936(5) & 0.0644(3) & 0.0732(14) \\ \text { H19A } & 0.7992 & 1.3479 & 0.0680 & 0.110^{*} \\ \text { H19B } & 0.6461 & 1.3648 & 0.0904 & 0.110^{*} \\ \text { H19C } & 0.6336 & 1.2561 & 0.0023 & 0.110^{*} \\ \text { C20 } & 0.8329(5) & 0.9635(6) & 0.2569(4) & 0.0837(15) \\ \text { H20A } & 0.8194 & 0.9630 & 0.3193 & 0.126^{*} \\ \text { H20B } & 0.9396 & 1.0446 & 0.2521 & 0.126^{*} \\ \text { H20C } & 0.8206 & 0.8624 & 0.2313 & 0.126^{*} \\ & & & & \end{array}$

Atomic displacement parameters $\left(\AA^{2}\right)$

\begin{tabular}{lllllll}
\hline & $U^{11}$ & $U^{22}$ & $U^{33}$ & $U^{12}$ & $U^{13}$ & $U^{23}$ \\
\hline Cd1 & $0.03665(17)$ & $0.05040(19)$ & $0.03889(17)$ & $0.02133(13)$ & $-0.00124(11)$ & $0.01172(12)$ \\
N1 & $0.0334(16)$ & $0.0441(18)$ & $0.0397(17)$ & $0.0198(15)$ & $0.0055(13)$ & $0.0101(14)$ \\
N2 & $0.0343(16)$ & $0.0515(19)$ & $0.0318(16)$ & $0.0219(15)$ & $0.0033(13)$ & $0.0070(13)$ \\
N3 & $0.056(2)$ & $0.103(3)$ & $0.0426(19)$ & $0.053(2)$ & $0.0011(16)$ & $-0.0061(19)$ \\
N4 & $0.0439(19)$ & $0.0461(19)$ & $0.0428(18)$ & $0.0198(17)$ & $0.0060(15)$ & $0.0060(14)$ \\
N5 & $0.0465(19)$ & $0.0442(18)$ & $0.0313(16)$ & $0.0218(16)$ & $0.0004(14)$ & $0.0064(13)$ \\
N6 & $0.047(2)$ & $0.072(3)$ & $0.064(2)$ & $0.026(2)$ & $-0.0057(17)$ & $0.0254(19)$ \\
N7 & $0.076(3)$ & $0.057(2)$ & $0.041(2)$ & $0.037(2)$ & $-0.0073(19)$ & $0.0058(17)$ \\
N8 & $0.0391(18)$ & $0.054(2)$ & $0.0418(18)$ & $0.0183(17)$ & $0.0073(15)$ & $0.0091(16)$
\end{tabular}




$\begin{array}{lllllll}\text { O1 } & 0.083(2) & 0.101(3) & 0.081(2) & 0.061(2) & 0.0066(19) & 0.0053(19) \\ \text { O2 } & 0.073(2) & 0.100(3) & 0.0541(19) & 0.044(2) & -0.0205(16) & -0.0151(17) \\ \text { O3 } & 0.111(3) & 0.096(3) & 0.0524(19) & 0.077(2) & -0.0252(18) & -0.0190(17) \\ \text { O4 } & 0.0460(16) & 0.0676(19) & 0.0608(18) & 0.0351(15) & 0.0044(13) & 0.0099(14) \\ \text { O5 } & 0.072(2) & 0.071(2) & 0.0607(18) & 0.0446(18) & 0.0102(15) & 0.0249(16) \\ \text { O6 } & 0.089(3) & 0.092(3) & 0.100(3) & 0.032(2) & 0.060(2) & 0.037(2) \\ \text { C1 } & 0.0311(18) & 0.0372(19) & 0.0331(19) & 0.0152(16) & 0.0029(15) & 0.0069(15) \\ \text { C2 } & 0.033(2) & 0.048(2) & 0.039(2) & 0.0206(18) & 0.0045(16) & 0.0052(17) \\ \text { C3 } & 0.034(2) & 0.055(2) & 0.049(2) & 0.0253(19) & -0.0001(17) & 0.0072(19) \\ \text { C4 } & 0.035(2) & 0.050(2) & 0.039(2) & 0.0176(18) & -0.0038(16) & 0.0045(17) \\ \text { C5 } & 0.0343(19) & 0.0355(19) & 0.0347(19) & 0.0139(17) & 0.0013(15) & 0.0042(15) \\ \text { C6 } & 0.041(2) & 0.039(2) & 0.037(2) & 0.0156(18) & 0.0013(16) & 0.0029(16) \\ \text { C7 } & 0.048(2) & 0.042(2) & 0.043(2) & 0.0206(19) & 0.0096(18) & 0.0027(17) \\ \text { C8 } & 0.0327(19) & 0.034(2) & 0.048(2) & 0.0130(16) & 0.0113(17) & 0.0103(17) \\ \text { C9 } & 0.062(3) & 0.076(3) & 0.037(2) & 0.036(2) & -0.0058(19) & -0.006(2) \\ \text { C10 } & 0.046(2) & 0.059(3) & 0.067(3) & 0.032(2) & 0.014(2) & 0.014(2) \\ \text { C11 } & 0.050(2) & 0.037(2) & 0.0300(19) & 0.0172(19) & 0.0063(17) & -0.0007(15) \\ \text { C12 } & 0.057(3) & 0.052(2) & 0.035(2) & 0.029(2) & 0.0013(18) & 0.0076(18) \\ \text { C13 } & 0.069(3) & 0.062(3) & 0.046(2) & 0.038(3) & 0.003(2) & 0.015(2) \\ \text { C14 } & 0.090(4) & 0.051(3) & 0.041(2) & 0.040(3) & 0.013(2) & 0.0157(19) \\ \text { C15 } & 0.067(3) & 0.038(2) & 0.034(2) & 0.023(2) & 0.0129(19) & 0.0019(17) \\ \text { C16 } & 0.075(3) & 0.040(2) & 0.036(2) & 0.016(2) & 0.014(2) & -0.0011(18) \\ \text { C17 } & 0.059(3) & 0.053(3) & 0.060(3) & 0.013(2) & 0.018(2) & 0.002(2) \\ \text { C18 } & 0.044(2) & 0.056(3) & 0.059(3) & 0.019(2) & 0.007(2) & 0.000(2) \\ \text { C19 } & 0.097(4) & 0.043(3) & 0.057(3) & 0.011(3) & 0.028(3) & 0.009(2) \\ \text { C20 } & 0.047(3) & 0.082(4) & 0.118(4) & 0.027(3) & 0.008(3) & 0.020(3)\end{array}$

Geometric parameters $\left(\AA,{ }^{\circ}\right)$

\begin{tabular}{llll}
\hline $\mathrm{Cd} 1-\mathrm{N} 5$ & $2.284(3)$ & $\mathrm{C} 4-\mathrm{H} 4$ & 0.9300 \\
$\mathrm{Cd} 1-\mathrm{O} 2$ & $2.355(3)$ & $\mathrm{C} 5-\mathrm{C} 6$ & $1.408(5)$ \\
$\mathrm{Cd} 1-\mathrm{N} 2$ & $2.361(3)$ & $\mathrm{C} 6-\mathrm{C} 7$ & $1.379(5)$ \\
$\mathrm{Cd} 1-\mathrm{O} 4$ & $2.433(3)$ & $\mathrm{C} 6-\mathrm{C} 9$ & $1.501(5)$ \\
$\mathrm{Cd} 1-\mathrm{N} 1$ & $2.448(3)$ & $\mathrm{C} 7-\mathrm{C} 8$ & $1.400(5)$ \\
$\mathrm{Cd} 1-\mathrm{N} 4$ & $2.586(3)$ & $\mathrm{C} 7-\mathrm{H} 7$ & 0.9300 \\
$\mathrm{~N} 1-\mathrm{C} 8$ & $1.326(4)$ & $\mathrm{C} 8-\mathrm{C} 10$ & $1.490(5)$ \\
$\mathrm{N} 1-\mathrm{C} 1$ & $1.359(4)$ & $\mathrm{C} 9-\mathrm{H} 9 \mathrm{~A}$ & 0.9600 \\
$\mathrm{~N} 2-\mathrm{C} 2$ & $1.328(4)$ & $\mathrm{C} 9-\mathrm{H} 9 \mathrm{~B}$ & 0.9600 \\
$\mathrm{~N} 2-\mathrm{C} 1$ & $1.356(4)$ & $\mathrm{C} 9-\mathrm{H} 9 \mathrm{C}$ & 0.9600 \\
$\mathrm{~N} 3-\mathrm{C} 2$ & $1.343(4)$ & $\mathrm{C} 10-\mathrm{H} 10 \mathrm{~A}$ & 0.9600 \\
$\mathrm{~N} 3-\mathrm{H} 3 \mathrm{~A}$ & 0.8600 & $\mathrm{C} 10-\mathrm{H} 10 \mathrm{~B}$ & 0.9600 \\
$\mathrm{~N} 3-\mathrm{H} 3 \mathrm{~B}$ & 0.8600 & $\mathrm{C} 10-\mathrm{H} 10 \mathrm{C}$ & 0.9600 \\
$\mathrm{~N} 4-\mathrm{C} 18$ & $1.339(5)$ & $\mathrm{C} 11-\mathrm{C} 15$ & $1.406(5)$ \\
$\mathrm{N} 4-\mathrm{C} 11$ & $1.342(5)$ & $\mathrm{C} 12-\mathrm{C} 13$ & $1.427(5)$ \\
$\mathrm{N} 5-\mathrm{C} 12$ & $1.333(4)$ & $\mathrm{C} 13-\mathrm{C} 14$ & $1.342(6)$ \\
$\mathrm{N} 5-\mathrm{C} 11$ & $1.364(5)$ & $\mathrm{C} 13-\mathrm{H} 13$ & 0.9300 \\
$\mathrm{~N} 6-\mathrm{C} 12$ & $1.330(5)$ & $\mathrm{C} 14-\mathrm{C} 15$ & $1.422(6)$ \\
$\mathrm{N} 6-\mathrm{H} 6 \mathrm{~A}$ & 0.8600 & $\mathrm{C} 14-\mathrm{H} 14$ & 0.9300
\end{tabular}




\begin{tabular}{|c|c|c|c|}
\hline N6-H6B & 0.8600 & $\mathrm{C} 15-\mathrm{C} 16$ & $1.406(6)$ \\
\hline $\mathrm{N} 7-\mathrm{O} 3$ & $1.229(4)$ & $\mathrm{C} 16-\mathrm{C} 17$ & $1.370(6)$ \\
\hline $\mathrm{N} 7-\mathrm{O} 1$ & $1.241(4)$ & $\mathrm{C} 16-\mathrm{C} 19$ & $1.514(5)$ \\
\hline $\mathrm{N} 7-\mathrm{O} 2$ & $1.250(4)$ & $\mathrm{C} 17-\mathrm{C} 18$ & $1.398(6)$ \\
\hline $\mathrm{N} 8-\mathrm{O} 6$ & $1.213(4)$ & $\mathrm{C} 17-\mathrm{H} 17$ & 0.9300 \\
\hline $\mathrm{N} 8-\mathrm{O} 5$ & $1.246(4)$ & $\mathrm{C} 18-\mathrm{C} 20$ & $1.498(6)$ \\
\hline $\mathrm{N} 8-\mathrm{O} 4$ & $1.250(4)$ & C19-H19A & 0.9600 \\
\hline $\mathrm{C} 1-\mathrm{C} 5$ & $1.405(4)$ & C19-H19B & 0.9600 \\
\hline $\mathrm{C} 2-\mathrm{C} 3$ & $1.423(5)$ & C19-H19C & 0.9600 \\
\hline $\mathrm{C} 3-\mathrm{C} 4$ & $1.351(5)$ & $\mathrm{C} 20-\mathrm{H} 20 \mathrm{~A}$ & 0.9600 \\
\hline $\mathrm{C} 3-\mathrm{H} 3$ & 0.9300 & $\mathrm{C} 20-\mathrm{H} 20 \mathrm{~B}$ & 0.9600 \\
\hline $\mathrm{C} 4-\mathrm{C} 5$ & $1.418(5)$ & $\mathrm{C} 20-\mathrm{H} 20 \mathrm{C}$ & 0.9600 \\
\hline $\mathrm{N} 5-\mathrm{Cd} 1-\mathrm{O} 2$ & $104.91(11)$ & $\mathrm{C} 7-\mathrm{C} 6-\mathrm{C} 9$ & $121.4(3)$ \\
\hline $\mathrm{N} 5-\mathrm{Cd} 1-\mathrm{N} 2$ & $140.76(11)$ & $\mathrm{C} 5-\mathrm{C} 6-\mathrm{C} 9$ & $121.2(3)$ \\
\hline $\mathrm{O} 2-\mathrm{Cd} 1-\mathrm{N} 2$ & $84.14(10)$ & $\mathrm{C} 6-\mathrm{C} 7-\mathrm{C} 8$ & $121.6(3)$ \\
\hline $\mathrm{N} 5-\mathrm{Cd} 1-\mathrm{O} 4$ & $130.94(10)$ & $\mathrm{C} 6-\mathrm{C} 7-\mathrm{H} 7$ & 119.2 \\
\hline $\mathrm{O} 2-\mathrm{Cd} 1-\mathrm{O} 4$ & $89.68(11)$ & $\mathrm{C} 8-\mathrm{C} 7-\mathrm{H} 7$ & 119.2 \\
\hline $\mathrm{N} 2-\mathrm{Cd} 1-\mathrm{O} 4$ & $86.30(9)$ & $\mathrm{N} 1-\mathrm{C} 8-\mathrm{C} 7$ & $121.4(3)$ \\
\hline $\mathrm{N} 5-\mathrm{Cd} 1-\mathrm{N} 1$ & $111.10(9)$ & $\mathrm{N} 1-\mathrm{C} 8-\mathrm{C} 10$ & $117.6(3)$ \\
\hline $\mathrm{O} 2-\mathrm{Cd} 1-\mathrm{N} 1$ & $139.39(10)$ & $\mathrm{C} 7-\mathrm{C} 8-\mathrm{C} 10$ & $121.0(3)$ \\
\hline $\mathrm{N} 2-\mathrm{Cd} 1-\mathrm{N} 1$ & $56.05(9)$ & $\mathrm{C} 6-\mathrm{C} 9-\mathrm{H} 9 \mathrm{~A}$ & 109.5 \\
\hline $\mathrm{O} 4-\mathrm{Cd} 1-\mathrm{N} 1$ & $80.82(9)$ & $\mathrm{C} 6-\mathrm{C} 9-\mathrm{H} 9 \mathrm{~B}$ & 109.5 \\
\hline $\mathrm{N} 5-\mathrm{Cd} 1-\mathrm{N} 4$ & $54.63(10)$ & $\mathrm{H} 9 \mathrm{~A}-\mathrm{C} 9-\mathrm{H} 9 \mathrm{~B}$ & 109.5 \\
\hline $\mathrm{O} 2-\mathrm{Cd} 1-\mathrm{N} 4$ & $91.13(11)$ & $\mathrm{C} 6-\mathrm{C} 9-\mathrm{H} 9 \mathrm{C}$ & 109.5 \\
\hline $\mathrm{N} 2-\mathrm{Cd} 1-\mathrm{N} 4$ & $87.67(10)$ & $\mathrm{H} 9 \mathrm{~A}-\mathrm{C} 9-\mathrm{H} 9 \mathrm{C}$ & 109.5 \\
\hline $\mathrm{O} 4-\mathrm{Cd} 1-\mathrm{N} 4$ & $173.80(9)$ & $\mathrm{H} 9 \mathrm{~B}-\mathrm{C} 9-\mathrm{H} 9 \mathrm{C}$ & 109.5 \\
\hline $\mathrm{N} 1-\mathrm{Cd} 1-\mathrm{N} 4$ & $94.61(9)$ & $\mathrm{C} 8-\mathrm{C} 10-\mathrm{H} 10 \mathrm{~A}$ & 109.5 \\
\hline $\mathrm{C} 8-\mathrm{N} 1-\mathrm{C} 1$ & $118.2(3)$ & $\mathrm{C} 8-\mathrm{C} 10-\mathrm{H} 10 \mathrm{~B}$ & 109.5 \\
\hline $\mathrm{C} 8-\mathrm{N} 1-\mathrm{Cd} 1$ & $148.2(2)$ & $\mathrm{H} 10 \mathrm{~A}-\mathrm{C} 10-\mathrm{H} 10 \mathrm{~B}$ & 109.5 \\
\hline $\mathrm{C} 1-\mathrm{N} 1-\mathrm{Cd} 1$ & $93.59(19)$ & $\mathrm{C} 8-\mathrm{C} 10-\mathrm{H} 10 \mathrm{C}$ & 109.5 \\
\hline $\mathrm{C} 2-\mathrm{N} 2-\mathrm{C} 1$ & $118.5(3)$ & $\mathrm{H} 10 \mathrm{~A}-\mathrm{C} 10-\mathrm{H} 10 \mathrm{C}$ & 109.5 \\
\hline $\mathrm{C} 2-\mathrm{N} 2-\mathrm{Cd} 1$ & $143.8(2)$ & $\mathrm{H} 10 \mathrm{~B}-\mathrm{C} 10-\mathrm{H} 10 \mathrm{C}$ & 109.5 \\
\hline $\mathrm{C} 1-\mathrm{N} 2-\mathrm{Cd} 1$ & $97.6(2)$ & $\mathrm{N} 4-\mathrm{C} 11-\mathrm{N} 5$ & $112.6(3)$ \\
\hline $\mathrm{C} 2-\mathrm{N} 3-\mathrm{H} 3 \mathrm{~A}$ & 120.0 & $\mathrm{~N} 4-\mathrm{C} 11-\mathrm{C} 15$ & $124.2(4)$ \\
\hline $\mathrm{C} 2-\mathrm{N} 3-\mathrm{H} 3 \mathrm{~B}$ & 120.0 & $\mathrm{~N} 5-\mathrm{C} 11-\mathrm{C} 15$ & $123.2(3)$ \\
\hline $\mathrm{H} 3 \mathrm{~A}-\mathrm{N} 3-\mathrm{H} 3 \mathrm{~B}$ & 120.0 & $\mathrm{~N} 6-\mathrm{C} 12-\mathrm{N} 5$ & $119.3(3)$ \\
\hline $\mathrm{C} 18-\mathrm{N} 4-\mathrm{C} 11$ & $117.4(3)$ & $\mathrm{N} 6-\mathrm{C} 12-\mathrm{C} 13$ & $120.0(4)$ \\
\hline $\mathrm{C} 18-\mathrm{N} 4-\mathrm{Cd} 1$ & $152.3(3)$ & $\mathrm{N} 5-\mathrm{C} 12-\mathrm{C} 13$ & $120.7(4)$ \\
\hline $\mathrm{C} 11-\mathrm{N} 4-\mathrm{Cd} 1$ & $89.8(2)$ & $\mathrm{C} 14-\mathrm{C} 13-\mathrm{C} 12$ & $120.4(4)$ \\
\hline $\mathrm{C} 12-\mathrm{N} 5-\mathrm{C} 11$ & $119.0(3)$ & $\mathrm{C} 14-\mathrm{C} 13-\mathrm{H} 13$ & 119.8 \\
\hline $\mathrm{C} 12-\mathrm{N} 5-\mathrm{Cd} 1$ & $137.6(3)$ & $\mathrm{C} 12-\mathrm{C} 13-\mathrm{H} 13$ & 119.8 \\
\hline $\mathrm{C} 11-\mathrm{N} 5-\mathrm{Cd} 1$ & $102.9(2)$ & $\mathrm{C} 13-\mathrm{C} 14-\mathrm{C} 15$ & $120.3(4)$ \\
\hline $\mathrm{C} 12-\mathrm{N} 6-\mathrm{H} 6 \mathrm{~A}$ & 120.0 & $\mathrm{C} 13-\mathrm{C} 14-\mathrm{H} 14$ & 119.8 \\
\hline $\mathrm{C} 12-\mathrm{N} 6-\mathrm{H} 6 \mathrm{~B}$ & 120.0 & $\mathrm{C} 15-\mathrm{C} 14-\mathrm{H} 14$ & 119.8 \\
\hline $\mathrm{H} 6 \mathrm{~A}-\mathrm{N} 6-\mathrm{H} 6 \mathrm{~B}$ & 120.0 & $\mathrm{C} 11-\mathrm{C} 15-\mathrm{C} 16$ & $117.8(4)$ \\
\hline $\mathrm{O} 3-\mathrm{N} 7-\mathrm{O} 1$ & $121.8(4)$ & $\mathrm{C} 11-\mathrm{C} 15-\mathrm{C} 14$ & $116.3(4)$ \\
\hline $\mathrm{O} 3-\mathrm{N} 7-\mathrm{O} 2$ & $120.9(4)$ & $\mathrm{C} 16-\mathrm{C} 15-\mathrm{C} 14$ & $125.9(4)$ \\
\hline
\end{tabular}




$\begin{array}{llll}\mathrm{O} 1-\mathrm{N} 7-\mathrm{O} 2 & 117.3(3) & \mathrm{C} 17-\mathrm{C} 16-\mathrm{C} 15 & 117.4(4) \\ \mathrm{O} 6-\mathrm{N} 8-\mathrm{O} 5 & 120.6(3) & \mathrm{C} 17-\mathrm{C} 16-\mathrm{C} 19 & 121.2(4) \\ \mathrm{O} 6-\mathrm{N} 8-\mathrm{O} 4 & 121.3(4) & \mathrm{C} 15-\mathrm{C} 16-\mathrm{C} 19 & 121.5(4) \\ \mathrm{O} 5-\mathrm{N} 8-\mathrm{O} 4 & 118.1(3) & \mathrm{C} 16-\mathrm{C} 17-\mathrm{C} 18 & 121.5(4) \\ \mathrm{N} 7-\mathrm{O} 2-\mathrm{C} 1 & 105.8(3) & \mathrm{C} 16-\mathrm{C} 17-\mathrm{H} 17 & 119.2 \\ \mathrm{~N} 8-\mathrm{O} 4-\mathrm{Cd} 1 & 100.6(2) & \mathrm{C} 18-\mathrm{C} 17-\mathrm{H} 17 & 119.2 \\ \mathrm{~N} 2-\mathrm{C} 1-\mathrm{N} 1 & 112.7(3) & \mathrm{N} 4-\mathrm{C} 18-\mathrm{C} 17 & 116.7(4) \\ \mathrm{N} 2-\mathrm{C} 1-\mathrm{C} 5 & 123.7(3) & \mathrm{N} 4-\mathrm{C} 18-\mathrm{C} 20 & 121.6(4) \\ \mathrm{N} 1-\mathrm{C} 1-\mathrm{C} 5 & 123.5(3) & \mathrm{C} 17-\mathrm{C} 18-\mathrm{C} 20 & 109.5 \\ \mathrm{~N} 2-\mathrm{C} 2-\mathrm{N} 3 & 118.1(3) & \mathrm{C} 16-\mathrm{C} 19-\mathrm{H} 19 \mathrm{~A} & 109.5 \\ \mathrm{~N} 2-\mathrm{C} 2-\mathrm{C} 3 & 121.8(3) & \mathrm{C} 16-\mathrm{C} 19-\mathrm{H} 19 \mathrm{~B} & 109.5 \\ \mathrm{~N} 3-\mathrm{C} 2-\mathrm{C} 3 & 120.1(3) & \mathrm{H} 19 \mathrm{~A}-\mathrm{C} 19-\mathrm{H} 19 \mathrm{~B} & 109.5 \\ \mathrm{C} 4-\mathrm{C} 3-\mathrm{C} 2 & 119.3(3) & \mathrm{C} 16-\mathrm{C} 19-\mathrm{H} 19 \mathrm{C} & 109.5 \\ \mathrm{C} 4-\mathrm{C} 3-\mathrm{H} 3 & 120.4 & \mathrm{H} 19 \mathrm{~A}-\mathrm{C} 19-\mathrm{H} 19 \mathrm{C} & 109.5 \\ \mathrm{C} 2-\mathrm{C} 3-\mathrm{H} 3 & 120.4 & \mathrm{H} 19 \mathrm{~B}-\mathrm{C} 19-\mathrm{H} 19 \mathrm{C} & 109.5 \\ \mathrm{C} 3-\mathrm{C} 4-\mathrm{C} 5 & 120.7(3) & \mathrm{C} 18-\mathrm{C} 20-\mathrm{H} 20 \mathrm{~A} & 109.5 \\ \mathrm{C} 3-\mathrm{C} 4-\mathrm{H} 4 & 119.7 & \mathrm{C} 18-\mathrm{C} 20-\mathrm{H} 20 \mathrm{~B} & 109.5 \\ \mathrm{C} 5-\mathrm{C} 4-\mathrm{H} 4 & 119.7 & \mathrm{H} 20 \mathrm{~A}-\mathrm{C} 20-\mathrm{H} 20 \mathrm{~B} & 109.5 \\ \mathrm{C} 1-\mathrm{C} 5-\mathrm{C} 6 & 117.8(3) & \mathrm{C} 18-\mathrm{C} 20-\mathrm{H} 20 \mathrm{C} & 109.5 \\ \mathrm{C} 1-\mathrm{C} 5-\mathrm{C} 4 & 116.0(3) & \mathrm{H} 20 \mathrm{~A}-\mathrm{C} 20-\mathrm{H} 20 \mathrm{C} & \\ \mathrm{C} 6-\mathrm{C} 5-\mathrm{C} 4 & 126.2(3) & \mathrm{H} 20 \mathrm{~B}-\mathrm{C} 20-\mathrm{H} 20 \mathrm{C} & \\ \mathrm{C} 7-\mathrm{C} 6-\mathrm{C} 5 & 117.4(3) & & \end{array}$

Hydrogen-bond geometry $\left(A,{ }^{\circ}\right)$

\begin{tabular}{lllll}
\hline$D-\mathrm{H} \cdots A$ & $D-\mathrm{H}$ & $\mathrm{H} \cdots A$ & $D \cdots A$ & $D-\mathrm{H} \cdots A$ \\
\hline $\mathrm{N} 3-\mathrm{H} 3 A \cdots \mathrm{O} 2$ & 0.86 & 2.23 & $3.033(4)$ & 155 \\
$\mathrm{~N} 3-\mathrm{H} 3 B \cdots \mathrm{O} 5^{\mathrm{i}}$ & 0.86 & 2.38 & $3.161(4)$ & 151 \\
$\mathrm{~N} 6-\mathrm{H} 6 A \cdots \mathrm{O} 5$ & 0.86 & 2.11 & $2.902(4)$ & 153 \\
$\mathrm{~N} 6-\mathrm{H} 6 B \cdots 3^{\text {ii }}$ & 0.86 & 2.18 & $2.979(4)$ & 154 \\
\hline
\end{tabular}

Symmetry codes: (i) $x+1, y, z$; (ii) $-x,-y+1,-z$. 